\title{
PRODUCCIÓN DE BRÓCOLI EN FUNCIÓN DEL GENOTIPO Y DOSIS DE NITRÓGENO
}

\section{BROCCOLI PRODUCTION BASED ON GENOTYPE AND NITROGEN DOSE}

\author{
Yurixhi A. Raya-Montaño, Patricio Apáez-Barrios, \\ Héctor Guillén-Andrade, Ma. Blanca Nieves Lara-Chavez*
}

Universidad Michoacana de San Nicolás de Hidalgo, Uruapan, Michoacán, México.

*Autor para correspondencia (chavez12001@yahoo.com.mx)

\section{RESUMEN}

El brócoli (Brassica oleracea var. Itálica) es una hortaliza que aporta vitaminas y elementos esenciales a la dieta humana, así como compuestos antioxidantes que mejoran la salud. Este cultivo puede ser alternativo en regiones productoras de aguacate (Persea americana), en donde las condiciones climáticas podrían ser adecuadas para el brócoli. Sin embargo se desconoce su comportamiento productivo, las variedades y dosis de fertilización nitrogenada más apropiados. El objetivo del presente estudio fue determinar la respuesta productiva y rentabilidad económica de las variedades de brócoli Monte Carlo y Green Magic con las dosis de 0,60 y $120 \mathrm{~kg} \mathrm{~N}^{-1}$ (N0, N60 y N120, respectivamente) en Uruapan, Michoacán, durante 2016. El ensayo se estableció en condiciones de campo a cielo abierto y con riego rodado, mediante un diseño de bloques al azar con cuatro repeticiones. Los resultados indican que Green Magic fue más productiva y mostró mayor respuesta $(P \leq 0.05)$ a la fertilización nitrogenada que Monte Carlo. Green Magic con N120 logró el máximo rendimiento, 14.1 t ha ${ }^{-1}$ y la más alta rentabilidad, $\$ 35,413.20 \mathrm{MXN}$. Monte Carlo con N0 y N60 y Green Magic con N0 no fueron económicamente rentables pues presentaron pérdidas económicas de hasta \$-48,714.2 MXN. Por lo tanto, la variedad Green Magic fertilizada con N120 es una opción de cultivo en Uruapan, Michoacán.

Palabras clave: Brassica oleracea, fertilización, nitrógeno, rentabilidad económica.

\section{SUMMARY}

Broccoli (Brassica oleracea var. Italica) is a vegetable that provides vitamins and essential elements to the human diet, as well as antioxidant compounds that improve health. This crop may be alternative in avocado (Persea americana) producing regions, where weather may be suitable for broccoli. However, its productive behavior, the most appropriate varieties and doses of nitrogen fertilization are unknown. The objective of this study was to determine the productive response and economic profitability of Monte Carlo and Green Magic broccoli varieties with the doses of 0,60 and $120 \mathrm{~kg} \mathrm{~N} \mathrm{ha}^{-1}$ (N0, N60 and N120, respectively) in Uruapan, Michoacán, in 2016. The trial was established in open field and irrigated field conditions. A randomized block design with four replications was used. It was found that Green Magic was more productive and showed greater response to nitrogen fertilization $(P \leq 0.05)$ than Monte Carlo. Green Magic with N120 achieved the highest yield, $14.1 \mathrm{t} \mathrm{ha}^{-1}$ and the highest profitability, $\$ 35,413.20 \mathrm{MXN}$. Monte Carlo with N0 and N60, as well as Green Magic with N0 were not economically profitable because of they had the highest economical losses, \$-48,714.2 MXN. Therefore, the cultivar Green Magic fertilized with $\mathrm{N} 120$ is an option to be grown in Uruapan, Michoacan.
Index words: Brassica oleracea, fertilization, nitrogen, economic profitability.

\section{INTRODUCCIÓN}

Actualmente, las inflorescencias del brócoli (Brassica oleracea var. Itálica) como producto fresco, congelado o deshidratado son ampliamente consumidas alrededor del mundo. Su consumo habitual provoca beneficios a la salud por la presencia de compuestos fenólicos y glucosinolatos, que tienen actividad antioxidante (Gratacós-Cubarsí et al., 2010; Bachiega et al., 2016). Existen diversas variedades mejoradas (Harrison Jr et al., 2015) que se han seleccionado para aumentar productividad, resistencia a plagas y enfermedades y adaptación a diferentes tipos de climas (Santoyo y Martínez, 2012).

El cultivo de brócoli en México está en auge (SIAP, 2018), debido a la promoción que se le ha dado como alimento funcional (Bachiega et al., 2016). Para el año 2016 la superficie destinada a este cultivo fue de 31,904 ha, que tuvieron un rendimiento medio de $15.9 \mathrm{t} \mathrm{ha}^{-1}$, por lo que produjeron $507,482 \mathrm{t}$, con un valor de $\$ 2,707$ millones de pesos mexicanos. Los principales estados productores son Guanajuato, Puebla y Michoacán, que en conjunto representan el 81 \% de la producción nacional. En Michoacán destacan los municipios de Tangancícuaro, Chilchota, Zamora y Purépero (SIAP, 2018). Existen municipios en Michoacán en donde no se reporta la siembra de brócoli, pero presenta algunas zonas con condiciones ambientales adecuadas para este cultivo (clima templado). Muchas de estas áreas tienen como principal cultivo al aguacate (Persea americana Mill) cv. Hass. Sin embargo, el aguacate al ser un monocultivo conlleva varios riesgos como pérdida de biodiversidad productiva y ecológica, inestabilidad de los mercados, crecimiento económico dependiente, etc. (De la Tejera, 2013). En este sentido, el brócoli podría ser una opción que ayudaría al productor aguacatero a 
diversificar sus cultivos. Sin embargo, para recomendar al brócoli como alternativa de cultivo es necesario conocer el manejo agronómico más apropiado en la zona.

La fertilización es determinante en el rendimiento y productividad de los cultivos. Dentro de los macronutrientes primarios, uno de los más limitantes es el nitrógeno $(\mathrm{N})(\mathrm{Xu}$ et al., 2012). La cantidad a aplicar va a depender del nivel inicial en el suelo, las características físicas y químicas del suelo, el rendimiento esperado, entre otras (Puenayan et al., 2010; Singh et al., 2015). Otro factor importante es determinar las variedades que mejor respondan a las condiciones medioambientales de la zona. Por lo anterior se planteó como objetivo: determinar la variedad de brócoli y la dosis de nitrógeno más apropiada que genere la mayor producción y rentabilidad económica en Uruapan Michoacán, como cultivo alternativo para los productores de esta región.

\section{MATERIALES Y MÉTODOS}

El estudio se estableció en la localidad de Jucutacato, Uruapan, Michoacán, localizada en las coordenadas $19^{\circ}$ $22^{\prime} 48^{\prime \prime}$ LN y $102^{\circ} 4^{\prime}$ '39" LO, a 1600 m de altitud. El clima del lugar es ACW, que corresponde a templado subhúmedo con abundantes lluvias en verano. Las temperaturas medias máximas son de $28{ }^{\circ} \mathrm{C}$ y mínimas de $12{ }^{\circ} \mathrm{C}$. La precipitación pluvial anual es de 1566 mm (García, 2004).

Para conocer el nivel inicial de fertilidad del suelo se realizó un análisis físico-químico, que indicó un $\mathrm{pH}$ de 6.5, con $7 \%$ de materia orgánica, $0.5 \%$ de $N$ total, 14 ppm de fósforo y CIC de 39 meq $100 \mathrm{~g}^{-1}$. La siembra del brócoli se realizó el 15 de junio de 2016 en charolas de poliestireno, previamente desinfectadas con inmersión por 5 min en una solución de agua con hipoclorito de sodio a la dosis de $5 \mathrm{~mL}$ $\mathrm{L}^{-1}$ y lavadas con agua limpia para eliminar el cloro. Las charolas se llenaron con peat moss y fueron colocadas bajo condiciones de invernadero. A los 45 días después de la siembra las plántulas fueron trasplantadas al suelo en campo a la densidad de población de 22,222 plantas ha ${ }^{-1}$. La distancia entre plantas fue de $0.5 \mathrm{~m}$ y $0.9 \mathrm{~m}$ entre surco.

Los factores y niveles evaluados fueron 2 variedades y 3 niveles de N. Las variedades de brócoli fueron Monte Carlo (M) y Green Magic (GM), que son de las más accesibles en el mercado. En ambas, las dosis de $\mathrm{N}$ evaluadas fueron $\mathrm{O}$, 60 y $120 \mathrm{~kg} \mathrm{ha}^{-1}$ (N0, N60 y N120, respectivamente). Los factores combinados generaron los tratamientos $\mathrm{M}-\mathrm{NO}$, M-N60, M-N120, GM-N0, GM-N60 y GM-N120, evaluados mediante el diseño experimental de bloques completos al azar con cuatro repeticiones. La unidad experimental estuvo formada por tres surcos de tres $m$ de longitud.

Además del N, a todos los tratamientos se les aplicaron
$40 \mathrm{~kg} \mathrm{ha}^{-1}$ de $\mathrm{P}_{2} \mathrm{O}_{5}$. Como fuentes de estos elementos se utilizó urea y fosfato diamónico. En los tratamientos con $\mathrm{N}$, la mitad de este elemento y todo el fósforo se aplicó 12 días después del trasplante (ddt) y el resto de $\mathrm{N}$ a los 42 ddt. El agua se suministró con riego rodado y se procuró siempre dejar a capacidad de campo.

A la madurez de cosecha se tomaron tres plantas como parcela útil de cada unidad experimental; en ellas se colectaron las pellas (floretes). En cada una de las pellas se midió el diámetro ecuatorial $(\mathrm{cm})$ con un vernier y se registró su peso en una báscula digital (kg). Con estos datos se determinó el diámetro y peso promedio de pellas por parcela. Con el número de pellas y la suma de los pesos de las pellas cosechadas por parcela útil se determinó el número de pellas por hectárea y el rendimiento de pellas $\left(\mathrm{kg} \mathrm{ha}^{-1}\right)$; para el calculó se consideró la densidad de población de 22,222 plantas ha-1.

Los datos de cada una de las variables se sometieron a un análisis de varianza con el paquete estadístico SAS versión 9.4 (SAS, 2017). A las variables con diferencias significativas se les aplicó la prueba de medias de Tukey al 5 $\%$ de probabilidad del error. Además se realizó un análisis económico para determinar la rentabilidad de cada tratamiento, mediante la ecuación: IN = YPy - ( $(\mathrm{XiPi}+\mathrm{CF})$ (Volke, 1982), donde IN: ingreso neto, Y: rendimiento (kg $\mathrm{ha}^{-1}$ ), Py: precio por kg, ¿XiPi: suma de costos variables (fertilizante, jornales para la cosecha, fletes etc.) y CF: costos fijos (preparación del terreno, semilla y jornales para el manejo del cultivo). El precio de venta considerado por kilogramo fue de $\$ 7.00 \mathrm{MXN}$. La tendencia de rendimiento por la combinación de dosis de $\mathrm{N}$ y variedad fue descrita por regresión.

\section{RESULTADOS Y DISCUSIÓN}

\section{Rendimiento de pellas y componentes del rendimiento}

El análisis de varianza mostró diferencias estadísticas altamente significativas $(P \leq 0.01)$ entre dosis de $\mathrm{N}$ y en la interacción Variedad $x$ Dosis de $\mathrm{N}$ en las variables rendimiento (RP), diámetro de pella (DP), peso promedio de pella (PP) y número de pellas (NP). Entre variedades sólo hubo diferencias estadísticas altamente significativas $(P \leq$ 0.01) en RP y PP (Cuadro 1).

De acuerdo con el análisis de medias (Cuadro 2), la variedad GM presentó un rendimiento de pellas $51.6 \%$ superior al de Monte Carlo $(P \leq 0.05)$, como consecuencia de que las pellas fueron $33 \%$ más pesadas $(P \leq 0.05)$, aunque en ambas variedades se observó similar cantidad y diámetro de las pellas. La aplicación de $\mathrm{N}$ incrementó el rendimiento de pellas (Cuadro 3). El mayor rendimiento se registró con 
Cuadro 1. Análisis de varianza de las variables evaluadas en variedades de brócoli y dosis de $\mathrm{N}$ en Uruapan, Michoacán, México en 2016.

\begin{tabular}{|c|c|c|c|c|}
\hline \multirow{2}{*}{ Variable } & \multicolumn{3}{|c|}{ Probabilidad de F } & \multirow{2}{*}{$C V(\%)$} \\
\hline & Variedad & Dosis de $\mathrm{N}$ & Variedad $\times$ Dosis de $\mathrm{N}$ & \\
\hline Rendimiento $\left(\mathrm{t} \mathrm{ha}^{-1}\right)$ & ** & ** & ** & 19.3 \\
\hline Diámetro (cm) & ns & ** & ** & 19.0 \\
\hline Peso promedio (g) & $\star \star$ & ** & ** & 18.7 \\
\hline Número de pellas & ns & ** & ** & 19.5 \\
\hline
\end{tabular}

$* *: P \leq 0.01, n s$ : no significativo. CV : coeficiente de variación.

N120 (P $\leq 0.05)$, que fue cinco y 1.6 veces mayor que con No y N60, respectivamente. Cabe destacar que con la dosis N120 se generó la mayor cantidad y peso de pellas ( $\mathrm{P} \leq$ 0.05). Estadísticamente las pellas fueron de similar diámetro con la dosis N60 y N120.

La respuesta a la dosis de $\mathrm{N}$ varió entre variedades. En la Figura 1 se presenta el análisis de regresión del rendimiento de pellas por cada variedad en función de la dosis de $\mathrm{N}$. Se observa que Green Magic mostró respuesta mayor a la fertilización nitrogenada. De acuerdo con el modelo de regresión lineal resultante, por cada kg de $\mathrm{N}$ aplicado Green Magic produjo $104.1 \mathrm{~kg}$ de pellas, mientras que Monte Carlo $70.2 \mathrm{~kg}$. En un mismo nivel de dosis de N, la variedad Green Magic superó a Monte Carlo. Con N120 y N60 Green Magic superó en rendimiento en 41.2 y $86.4 \%$ al de Monte Carlo (Cuadro 4). Este comportamiento fue debido a que las pellas fueron más pesadas. En ambas variedades, con NO las plantas de brócoli mostraron el rendimiento más bajo, como consecuencia de que una cantidad considerable de plantas no desarrollaron pella, únicamente follaje (Cuadro 4).

En el presente estudio, el aumento en el RP con la aplicación de $\mathrm{N}$ fue provocado porque este elemento incrementó la cantidad de plantas que produjeron pellas; además estas fueron de mayor diámetro y peso. Respuesta similar se encontró en la variedad Maratón y el híbrido Legacy (Yoldas et al., 2008; Puenayan et al., 2010). Se menciona que es importante producir pellas de grandes diámetros debido a que contribuyen a incrementar el rendimiento final y con ello generar mayor rentabilidad (Singh et al., 2015; Maldonado-Montalvo et al., 2017). El efecto positivo de la aplicación de $\mathrm{N}$ sobre los componentes del rendimiento y $\mathrm{RP}$ en el presente estudio se atribuye al bajo nivel inicial de $\mathrm{N}$ en el suelo $(0.5 \%$ ), que se incrementó con la fertilización que pudo haber favorecido la actividad fotosintética (Apáez et al., 2014) y con ellos la acumulación de fotosintatos, muchos de los cuales son destinados al órgano de interés económico, en este caso la pella (inflorescencia).
En este estudio la variedad Green Magic fertilizada con N120 generó el mayor RP (14.1 t ha-1), que está dentro del rango de rendimiento de brócoli reportado para las regiones productoras de México (11 a $21 \mathrm{t} \mathrm{ha}^{-1}$ ) (Muramoto et al., 2011; Santoyo y Martínez, 2012). De acuerdo con Toivonen et al. (1994), la aplicación de $120 \mathrm{~kg} \mathrm{~N} \mathrm{ha}^{-1}$ se considera una dosis moderada de fertilización, ya que se han encontrado rendimientos mayores con dosis más elevadas. Al respecto, Puenayan et al. (2010), con el híbrido Legacy fertilizado con $150 \mathrm{~kg} \mathrm{~N} \mathrm{ha}^{-1}$, lograron un rendimiento de $28.7 \mathrm{t} \mathrm{ha}^{-1}$, que es superior al máximo obtenido en el presente estudio. Yoldas et al. (2008), con la variedad Maratón, obtuvieron 32.8 y 34.6 t ha $^{-1}$ con 150 y $300 \mathrm{~kg} \mathrm{~N} \mathrm{ha}^{-1}$, respectivamente. Mientras que Zebarth et al. (1995) encontraron que con la dosis de $400 \mathrm{~kg} \mathrm{~N} \mathrm{ha}^{-1}$ obtuvieron un rendimiento de $20 \mathrm{t}$ $\mathrm{ha}^{-1}$ en la variedad Emperor. Esta variación en la dosis óptima se explica porque la cantidad de $\mathrm{N}$ más apropiada está en función del nivel inicial de $\mathrm{N}$ en el suelo, las características físicas y químicas del suelo, el clima, el rendimiento esperado y la densidad de población (Zebarth et al., 1995).

Se ha encontrado que los máximos rendimientos en brócoli son frecuentemente obtenidos con altas tasas de N. Sin embargo, la aplicación de dosis elevadas de este elemento reduce el porcentaje de $\mathrm{N}$ recuperado por el cultivo, que resulta en la acumulación de $\mathrm{N}$ inorgánico residual en el suelo. Esto representa un riesgo ambiental porque se puede lixiviar como nitrato y contaminar el agua subterránea con graves consecuencias a la salud humana al beber este tipo de agua (Zebarth et al., 1995). Por lo tanto, es importante conocer este tipo de aspectos para hacer recomendaciones de dosis de fertilizantes, que equilibren el valor del cultivo con el riesgo ambiental.

\section{Análisis de rentabilidad económica}

El análisis económico por tratamiento se describe en el Cuadro 5. Se observa que los costos variables y totales más elevados se generaron con la dosis N120 de fertilización. Sin embargo, con estos se lograron los mayores 
Cuadro 2. Promedios por variedades de brócoli de las variables evaluadas en Uruapan, Michoacán, México en 2016.

\begin{tabular}{lcccc}
\hline Variedad & Rendimiento $\left(\mathrm{t} \mathrm{ha}^{-1}\right)$ & Diámetro $(\mathrm{cm})$ & Peso promedio $(\mathrm{g})$ & Número de pellas por ha \\
\hline Monte Carlo & $5.58 \mathrm{~b}$ & $16.23 \mathrm{a}$ & $0.36 \mathrm{~b}$ & $14,480 \mathrm{a}$ \\
Green Magic & $8.46 \mathrm{a}$ & $16.33 \mathrm{a}$ & $0.48 \mathrm{a}$ & $15,918 \mathrm{a}$ \\
DSH $(0.05)$ & 2.3 & 6.34 & 0.11 & $3,047.1$
\end{tabular}

Medias con letras iguales dentro de cada columna no son diferentes estadísticamente (Tukey, P $\leq 0.05)$. DSH : diferencia significativa honesta.

Cuadro 3. Promedios por dosis de N de las variables evaluadas en Uruapan, Michoacán, México en 2016.

\begin{tabular}{lcccc}
\hline Dosis de $\mathrm{N}\left(\mathrm{kg} \mathrm{ha}^{-1}\right)$ & Rendimiento $\left(\mathrm{t} \mathrm{ha}^{-1}\right)$ & Diámetro $(\mathrm{cm})$ & Peso promedio $(\mathrm{g})$ & Número de pellas por ha \\
\hline 0 & $1.62 \mathrm{c}$ & $14.98 \mathrm{~b}$ & $0.24 \mathrm{c}$ & $7,552 \mathrm{c}$ \\
60 & $7.36 \mathrm{~b}$ & $16.44 \mathrm{a}$ & $0.41 \mathrm{~b}$ & $17,791 \mathrm{~b}$ \\
120 & $12.08 \mathrm{a}$ & $17.42 \mathrm{a}$ & $0.60 \mathrm{a}$ & $20,256 \mathrm{a}$ \\
$\mathrm{DSH}(0.05)$ & 1.73 & 1.25 & 0.12 & $1,859.2$ \\
\hline
\end{tabular}

Medias con letras iguales dentro de cada columna no son diferentes estadísticamente (Tukey, P $\leq 0.05$ ). DSH : diferencia significativa honesta.

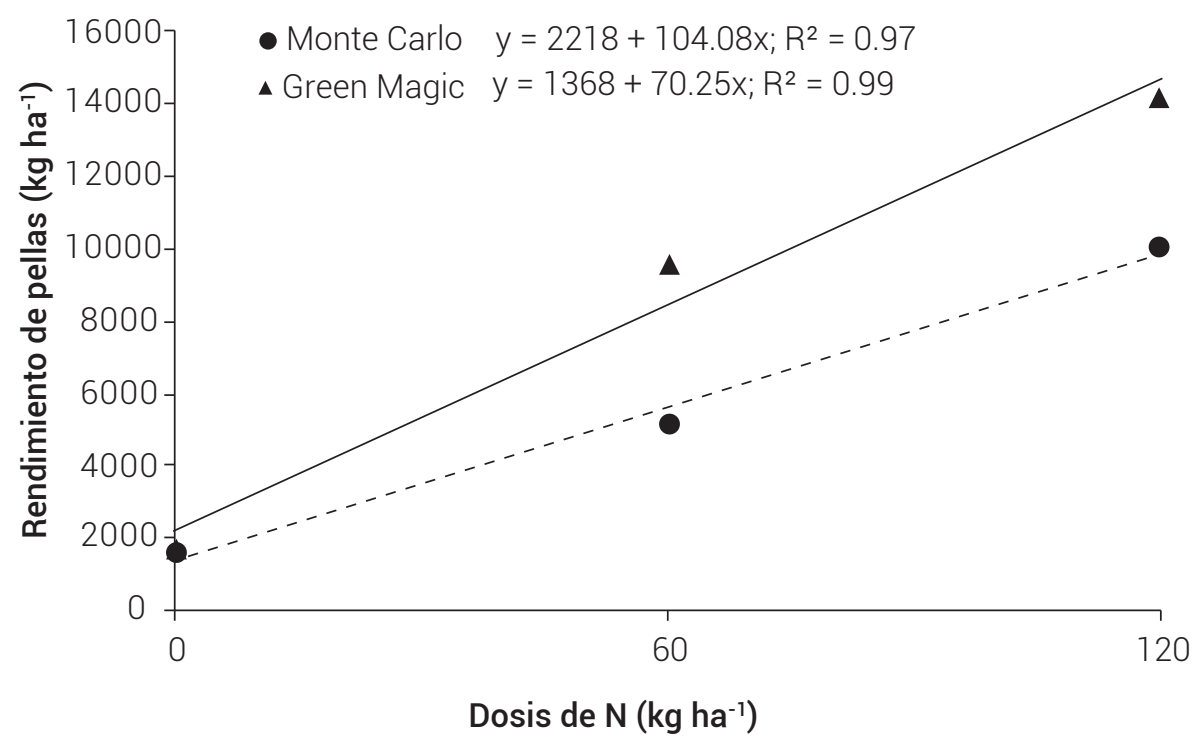

Figura 1. Análisis de regresión del rendimiento de pellas del brócoli en función de la variedad y dosis de nitrógeno.

Cuadro 4. Promedios de las variables evaluadas en la combinación variedades y dosis de $\mathrm{N}$ en Uruapan, Michoacán, México en 2016.

\begin{tabular}{lccccc}
\hline Variedad & Dosis de N & Rendimiento $\left(\mathrm{t} \mathrm{ha}^{-1}\right)$ & Diámetro $(\mathrm{cm})$ & Peso promedio $(\mathrm{g})$ & Número de pellas por ha \\
\hline \multirow{3}{*}{ Monte Carlo } & 0 & $1.59 \mathrm{~d}$ & $16.31 \mathrm{a}$ & $0.25 \mathrm{~b}$ & $6988 \mathrm{c}$ \\
& 60 & $5.14 \mathrm{c}$ & $15.62 \mathrm{~b}$ & $0.30 \mathrm{~b}$ & $17,272 \mathrm{~b}$ \\
& 120 & $10.02 \mathrm{~b}$ & $16.75 \mathrm{a}$ & $0.53 \mathrm{a}$ & $19181 \mathrm{a}$ \\
Green Magic & 0 & $1.66 \mathrm{~d}$ & $13.65 \mathrm{c}$ & $0.22 \mathrm{~b}$ & $8115 \mathrm{c}$ \\
& 60 & $9.58 \mathrm{~b}$ & $17.26 \mathrm{a}$ & $0.53 \mathrm{a}$ & $18,309 \mathrm{a}$ \\
DSH (0.05) & 120 & $14.15 \mathrm{a}$ & $18.09 \mathrm{a}$ & $0.68 \mathrm{a}$ & $21,4331 \mathrm{a}$ \\
\hline
\end{tabular}

Medias con letras iguales dentro de cada columna no son diferentes estadísticamente (Tukey, P $\leq 0.05)$. DSH : diferencia significativa honesta. 
Cuadro 5. Costos de producción y rentabilidad del cultivo de brócoli en Uruapan, Michoacán, México en 2016.

\begin{tabular}{|c|c|c|c|c|c|c|c|c|}
\hline \multirow{2}{*}{ Variedad } & Dosis & Rendimiento & IT & CF & CV & CT & IN & GPI \\
\hline & $\mathrm{kg} \mathrm{N} \mathrm{ha}^{-1}$ & $\mathrm{~kg} \mathrm{ha}^{-1}$ & \multicolumn{6}{|c|}{ - - - - - - - - - - - - - - \$ - - - - - - - - - - - - - } \\
\hline \multirow[t]{3}{*}{ Monte Carlo } & 0 & 3633 & 25,431 & 27,350 & $32,970.1$ & $60,320.14$ & $-34,889.1$ & -0.58 \\
\hline & 60 & 5140 & 35,980 & 27,350 & $35,870.0$ & $63,219.97$ & $-27,240.0$ & -0.43 \\
\hline & 120 & 10,016 & 70,112 & 27,350 & $36,293.8$ & $63,643.84$ & 6468.2 & 0.10 \\
\hline \multirow[t]{3}{*}{ Green Magic } & 0 & 1658 & 11,606 & 27,350 & $32,970.1$ & $60,320.14$ & $-48,714.1$ & -0.81 \\
\hline & 60 & 9582 & 67,074 & 27,350 & $35,870.0$ & $63,219.97$ & 3854.0 & 0.06 \\
\hline & 120 & 14,151 & 99,057 & 27,350 & $36,293.8$ & $63,643.84$ & $35,413.2$ & 0.56 \\
\hline
\end{tabular}

IT: Ingreso total (rendimiento * precio por kg de pella (\$7.00)). CF: Costo fijo (incluye costos de preparación del terreno, semilla, jornales para el manejo del cultivo). CV: Costos variables (incluyen el costo de fertilizante, cosecha y flete). CT: Costo total (costo fijo + costo variable). IN: Ingreso neto (ingreso total - costo total). GPI: ganancia por peso invertido.

rendimientos e ingresos totales. Debido a esto se amortizaron los costos totales, de tal manera que presentaron el más alto ingreso neto. Con la variedad Green Magic con N120 se logró obtener la ganancia económica más alta, que superó en \$28,945.0 al obtenido con Monte Carlo con N120. Así mismo, con este tratamiento se obtuvo una ganancia por peso invertido de 0.56 , superior a lo logrado con los otros tratamientos. Cabe destacar que ambas variedades, con N0 y Monte Carlo con N60, no son económicamente rentables.

\section{CONCLUSIONES}

La variedad Green Magic presentó el mayor rendimiento y peso promedio de pellas que Monte Carlo. La aplicación de $\mathrm{N}$ generó el rendimiento más alto de pellas, por lo que Green Magic fertilizada con la dosis de $120 \mathrm{~kg} \mathrm{ha}^{-1}$ de N registró el rendimiento más elevado, pero también un ingreso neto y ganancia por peso invertido superior a los otros tratamientos.

\section{BIBLIOGRAFIA}

Apáez B. P., E. J. A. S. Escalante, G. M. T. Rodríguez, C. M. C. González and M. Apáez (2014) Analysis of Cowpea Growth and Production in Maize Trellis with Nitrogen and Phosphorus. International Journal of AgriScience 4:102-108.

Bachiega P., J. M. Salgado, J. E. de Carvalho, A. L. T. Ruiz, K. Schwarz, T. Tezotto and M. Morzelle (2016) Antioxidant and antiproliferative activities in different maturation stages of broccoli (Brassica oleracea Italica) biofortified with selenium. Food chemistry 190:771-776, https://doi.org/10.1016/j.foodchem.2015.06.024

De la Tejera H. B., A. S. Ocampo, H. Santamaría, T. G. Morales y C. Olivares (2013) El oro verde en Michoacán: ¿un crecimiento sin fronteras? Acercamiento a la problemática y retos del sector aguacatero para el Estado y la sociedad. Economía y Sociedad 17:1540, http://www.redalyc.org/articulo.oa?id=51030120002

García E (2004) Modificación al sistema de clasificación climática de
Köppen. México: Instituto de Geografía, Universidad Nacional Autónoma de México. 90 p

Gratacós-Cubarsí M., A. Ribas-Agusti, J. A. García-Regueiro and M. Castellari (2010) Simultaneous evaluation of intact glucosinolates and phenolic compounds by UPLC-DAD-MS/MS in Brassica oleracea L. var. botrytis. Food Chemistry 121:257-263, https://doi. org/10.1016/j.foodchem.2009.11.081

Harrison Jr, H. F., M. W. Farnham and D. M. Jackson (2015) Tolerance of broccoli cultivars to pre-transplanting clomazone. Crop Protection 69:28-33, https://doi.org/10.1016/j.cropro.2014.11.013

Maldonado-Montalvo J., J. Ramírez-Juárez, J. A. Méndez-Espinoza y N. Pérez-Ramírez (2017) El sistema de producción del brócoli desde la perspectiva del campo social de Pierre Bourdieu. Estudios sociales 27:1-10, http://dx.doi.org/10.24836/es.v27i5.487

Muramoto J., R. F. Smith, C. Shennan, K. M. Klonsky, J. Leap, M. S. Ruiz and S. R. Gliessman (2011) Nitrogen contribution of legume/cereal mixed cover crops and organic fertilizers to an organic broccoli crop. Hortscience 46:1154-1162.

Puenayan I. A., R. F. Córdoba y S. A. Unigarro (2010) Respuesta del brócoli Brassica oleracea var. Italica L. Híbrido Legacy a la fertilización con N - P - K en el municipio de Pasto, Nariño. Revista de Ciencias Agrícolas 27:49-57.

Santoyo J. J. A. y A. C. 0. Martínez (2012) Tecnología de producción del brocoli: Fundación PRODUCE Sinaloa A. C. Innovación y progreso. Sinaloa. 29 p.

SIAP, Servicio de Información Agroalimentaria y Pesquera (2018) Anuario Estadístico de la Producción Agrícola. Servicio de Información Agroalimentaria y Pesquera. SAGARPA. Cd. de México. http:// nube.siap.gob.mx/cierre agrícola/ (Agosto 2018).

SAS Institute (2017) SAS 9.4 Companion for Windows. 5th ed. SAS Institute Inc. Cary, NC, USA. 700 p.

Singh M. K., T. Chand, M. Kumar, K. V. Singh, S. K. Lodhi, V. P. Singh and V. S. Sirohi (2015) Response of different doses of NPK and boron on growth and yield of broccoli (Brassica oleracea L. var. italica). International Journal of Bio-resource Stress Management 6:108-112, http://doi.org/10.5958/0976-4038.2015.00016.0

Toivonen P. M., B. J. Zebarth and P. A. Bowen (1994) Effect of nitrogen fertilization on head size, vitamin $\mathrm{C}$ content and storage life of broccoli (Brassica oleracea var. italica). Canadian journal of plant science 74:607-610, https://doi.org/10.4141/cjps94-109

Volke H. V. (1982) Optimización de insumos de la producción en la agricultura. Colegio de Postgraduados. Montecillo, Estado de México. 61 p.

Xu G., X. Fan and A. J. Miller (2012) Plant nitrogen assimilation and use efficiency. Annual review of plant biology 63:153-182, https:// doi.org/10.1146/annurev-arplant-042811-105532 
Yoldas F., S. Ceylan, B. Yagmur and N. Mordogan (2008) Effects of nitrogen fertilizer on yield quality and nutrient content in broccoli. Journal of Plant Nutrition 31:1333-1343, https://doi. org/10.1080/01904160802135118
Zebarth B. J., P. A. Bowen and P. M. A Toivonen (1995) Influence of nitrogen fertilization on broccoli yield, nitrogen accumulation and apparent fertilizer-nitrogen recovery. Canadian Journal of Plant Science 75:717-725, https://doi.org/10.4141/cjps95-122 